\title{
Erratum to: $\mathbf{P} 2$ receptors in atherosclerosis and postangioplasty restenosis
}

\author{
Cheikh I. Seye ${ }^{1}$ - Qiongman Kong ${ }^{1}$ - Ningpu Yu ${ }^{1}$ • Fernando A. Gonzalez ${ }^{2}$. \\ Laurie Erb ${ }^{1} \cdot$ Gary A. Weisman ${ }^{1}$
}

Published online: 31 July 2015

(C) Springer Science+Business Media Dordrecht 2015

Erratum to: Purinergic Signalling (2006) 2(3):471-480

DOI 10.1007/s11302-006-9015-1

This article was unintentionally published twice in this journal.

Following should be considered the version of record and used for citation purposes:

"Cheikh I. Seye, Qiongman Kong, Ningpu Yu, Fernando A. Gonzalez, Laurie Erb \& Gary A. Weisman, $\mathrm{P} 2$ receptors in atherosclerosis and postangioplasty restenosis, Purinergic Signalling, Volume 2, Issue 3, pages 471-480, 10.1007/s11302-006-9015-1".

The duplicate "Cheikh I. Seye, Qiongman Kong, Ningpu Yu, Fernando A. Gonzalez, Laurie Erb \& Gary A. Weisman, P2 receptors in atherosclerosis and postangioplasty restenosis, Purinergic Signalling, Volume 3, Issues 1-2, pages 153-162, 10.1007/s11302006-9047-6" is to be ignored.

The publisher apologizes to the readers of the journal for not detecting the duplication during the publication process.

The online version of the original article can be found at http://dx.doi.org/ 10.1007/s11302-006-9015-1.

Cheikh I. Seye

seyec@missouri.edu

1 Department of Biochemistry, 540C Life Sciences Center,

University of Missouri-Columbia,

1201 Rollins Road,

Columbia, MO 65211-7310, USA

2 Department of Chemistry, Rio Piedras Campus,

University of Puerto-Rico,

San Juan, PR 00931, USA 